\title{
Innovaciones en la evaluación de los problemas emocionales en niños y adolescentes: Presentación del número monográfico
}

\author{
David Pineda \\ Universidad Miguel Hernández de Elche, Elche, España
}

Los problemas emocionales presentan una elevada tasa de prevalencia en la población de niños y adolescentes. En los últimos años se ha avanzado de forma significativa en el conocimiento de la psicopatología y los trastornos emocionales que se dan durante la infancia y la adolescencia. Así mismo, también se ha producido un notable progreso en el tratamiento de estos problemas y/o trastornos, con el desarrollo de nuevos enfoques terapéuticos basados en la terapia cognitivo-conductual, diseñados para ser aplicados en el ámbito infanto-juvenil, tales como los nuevos protocolos basados en el enfoque transdiagnóstico de la terapia cognitivo-conductual. Sin embargo, se ha prestado una menor atención a la evaluación psicológica, cuyo progreso constituye una base necesaria para el avance, tanto de la psicopatología como del tratamiento de los problemas y trastornos emocionales. La evaluación psicológica basada en la evidencia constituye un aspecto fundamental en psicología clínica, y un pilar necesario tanto para la psicopatología como para el tratamiento de los trastornos emocionales. Sin duda, la evaluación psicológica ha adquirido recientemente un papel relevante en el campo de los problemas y los trastornos emocionales. La proliferación y validación empírica de nuevos instrumentos de evaluación en este campo y su posible proyección como herramientas de elevada utilidad para la investigación y tratamiento de las alteraciones emocionales motivó la publicación del presente número monográfico, en el cual han participado algunos de los equipos más representativos en el campo de la evaluación infanto-juvenil.

En el primer artículo se describe un estudio liderado por la profesora Salvador de la Universidad de Coimbra (Portugal), en el que participan colaboradores de cuatro países (Portugal, UK, USA y Canadá), mediante el cual se lleva a cabo una validación del Test Anxiety Acceptance and Action Questionnaire for Adolescents (TA-
AAQ-A). La adaptación de la prueba ha mostrado unas buenas propiedades psicométricas, con un adecuado ajuste y demostración de su invarianza factorial, basándose en una muestra de 827 adolescentes de 10 centros educativos de Portugal. Esta nueva herramienta aporta una medida sobre la flexibilidad cognitiva en situaciones de evaluación (exámenes).

En no pocas ocasiones se adaptan versiones de cuestionario de lápiz y papel de diferentes escalas para ser aplicadas apoyándose en las posibilidades que proporcionan las las nuevas tecnologías. Sin embargo, aunque estas adaptaciones suelen ser de utilidad, no están específicamente pensadas para ser aplicadas online. Otra limitación que nos encontramos en el campo es que tenemos muchas escalas específicas para la detección de determinados cuadros sintomatológicos, algunas incluso abarcan varios de estos, pero pocas permiten evaluar el espectro de los problemas emocionales. El estudio llevado a cabo por investigadores del grupo AITANA de la Universidad Miguel Hernández de Elche, presenta datos sobre la validez diagnóstica de las Escala DetectaWeb-Malestar, una herramienta diseñada para el screening de los problemas emocionales que permite evaluar de forma rápida y precisa, a través de una plataforma online, síntomas de ansiedad, depresión, tendencia al suicidio, estrés postraumático y trastorno obsesivo-compulsivo.

La escala ha demostrado ser eficaz en la detección y diagnóstico de los principales trastornos emocionales en niños y adolecentes.

La impulsividad, al igual que sucede con la ira o la irritabilidad, está íntimamente relacionada con los problemas emocionales y con los déficits en la regulación de las emociones. Se trata de un constructo multidimensional que abarca comportamientos relacionados con la falta de control en la inhibición de respuesta, la falta de planificación y la preferencia por recompensas inmedia- 
tas, aunque pequeñas, frente a otras más grandes pero demoradas. Los comportamientos impulsivos podrían explicar parcialmente el uso, el abuso y las adicciones, tanto a sustancias como comportamentales. Por ello, resulta necesario disponer de herramientas que evalúen este constructo en sus diferentes dimensiones. Un grupo de investigadores de Argentina (Universidad Nacional de Córdoba, Instituto de Investigaciones Psicológicas, e Instituto de Investigación Médica) presentan datos sobre la adaptación y validación de la versión breve de la Escala de Impulsividad para Niños y Adolescentes. Los datos reportados por los autores muestran evidencias de validez, fiabilidad e invarianza factorial entre sexos. Los autores concluyen que la versión argentina de esta escala es un instrumento adecuado para la evaluación de la impulsividad en sus distintas dimensiones.

Entre los múltiples escenarios que pueden darse durante el periodo de desarrollo de la niñez y la adolescencia, la ruptura del núcleo familiar es uno de los que tienen un impacto más negativo en el funcionamiento de los niños, y en menor medida de los adolescentes. Parte de ese impacto está regulado por las creencias y asunciones que los niños tengan sobre la ruptura del núcleo de convivencia. Además, el avance de las sociedades occidentales está llevando aparejado un aumento del número de divorcios, alcanzando cifras de considerable envergadura. Este aumento de los casos de separación, unido al impacto que las creencias sobre estos procesos tienen en los hijos, hacen necesario el desarrollo de instrumentos de evaluación que nos permitan explorar y valorar la presencia de estas creencias, y sobretodo aquellas más relacionadas con la asignación de culpas entre los miembros del grupo familiar, incluido el culparse a sí mimos, las creencias sobre la exclusión social que entre sus iguales pueda suponer esta separación, el miedo al abandono o la esperanza de reunificación. Los profesores José Pedro Espada y Mireia Orgilés del grupo AITANA lideran un estudio sobre la validación de la versión española de la Children's Beliefs About Parental Divorce Scale (CBAPS). Este trabajo supone un importante avance en la valoración de las creencias problemáticas asociadas al proceso de ruptura familiar. Tiene, además, la ventaja de su aplicabilidad en diversos ámbitos, incluyendo tanto al contexto clínico como el de la evaluación forense.

Otro importante aspecto relacionado con el ajuste psicológico infantil es el rechazo parental. El profesor Miguel Ángel Carrasco (Universidad Nacional de Educación a Distancia), experto reconocido en el campo de la evaluación infanto-juvenil, nos aporta resultados sobre el efecto moderador de la percepción que tienen los niños sobre el ambiente familiar, desde una perspectiva multi-informante, en la relación entre el rechazo paren- tal y el ajuste psicológico infantil. Los resultados que se presentan en este artículo muestran diferencias en la asociación entre el rechazo parental y el ajuste, condicionadas por la fuente informante. Además, la percepción subjetiva que tiene el menor sobre el afecto contextual moderaba el efecto del rechazo materno sobre los problemas exteriorizados informados por el padre. Los autores destacan la necesidad de incluir tanto a los propios menores como a sus padres en la evaluación de las relaciones familiares y el ajuste psicológico infantil.

El equipo de la profesora Concepción López-Soler (Universidad de Murcia) describe un interesante trabajo en el que hacen una comparación de diferentes criterios diagnósticos para el trastorno de estrés postraumático (TEPT) en niños y adolescentes. Este trabajo pone de manifiesto la necesidad de revisar los criterios preponderantes en el panorama clínico actual, donde no parece que se esté teniendo en cuenta la necesidad de establecer unos criterios diagnósticos sensibles con respecto al desarrollo evolutivo de los menores. Como consecuencia de esta falta de sensibilidad en dichos criterios, nos encontramos con una tasa de infradiagnóstico notable. Siendo el diagnostico del TEPT fundamental para el abordaje terapéutico, resulta perentorio contar con herramientas de evaluación, no solo validas y fiables, sino también que presenten una adecuada sensibilidad y especificidad.

Finalmente, la contribución de la profesora Esther Calvete de la Universidad Deusto (Bilbao) ha versado sobre la validación de la versión breve del Young-3 Schemas Questionnaire (YSQ-3-B), una importante herramienta para la evaluación de los esquemas desadaptativos tempranos en adolescentes y jóvenes. Estos esquemas tempranos se han asociado a numerosos problemas psicológicos como la depresión o la ansiedad entre otros. Se trata de un cuestionario de 54 ítems que evalúan los 18 esquemas desadaptativos tempranos, agrupados en torno a cinco dominios como son el dominio de desconexión y rechazo; el dominio de autonomía y ejecución dañinos; el dominio de límites dañinos; el dominio de foco en los demás; y por último el dominio de sobrevigilancia e inhibición. La herramienta está orientada para utilizarse en contestos clínicos de intervención.

En resumen, el monográfico aglutina importantes aportaciones en la evaluación de los problemas emocionales en niños y adolescentes, con relevantes implicaciones teóricas y prácticas en el campo de la evaluación tanto clínica como forense. Esperamos que resulten de interés para nuestros lectores, supongan una aportación relevante para la práctica diaria de la evaluación basada en la evidencia y fomenten de algún modo el avance de la investigación en el campo de la evaluación. 